\title{
ANISOTROPIC CONSTITUTIVE RELATIONSHIPS IN ENERGETIC MATERIALS: PETN AND HMX
}

\author{
M. Conroy ${ }^{1}$, I.I. Oleynik ${ }^{1}$, S.V. Zybin' ${ }^{2}$, and C. T. White ${ }^{3}$ \\ ${ }^{1}$ Department of Physics, University of South Florida, Tampa, FL 33620 \\ ${ }^{2}$ Materials and Process Simulation Center, California Institute of Technology, Pasadena, CA 91125 \\ ${ }^{3}$ Naval Research Laboratory, Washington, DC 20375
}

\begin{abstract}
This paper presents results of first-principles density functional calculations of the equation of state (EOS) of PETN-I and $\beta$-HMX. The isotropic EOS for hydrostatic compression has been extended to include uniaxial compressions in the [100], [010], [001], [110], [101], [011], and [111] directions up to compression ratio $\mathrm{V} / \mathrm{V}_{0}=0.70$. Equilibrium properties, including lattice parameters and elastic constants, as well as hydrostatic EOS are in good agreement with available experimental data. The shear stresses of uniaxially compressed PETN-I and $\beta$-HMX have been evaluated and their behavior as a function of compression ratio has been used to make predictions of shock sensitivity of these EMs. A comparison of predicted sensitivities with available experimental data has also been performed.
\end{abstract}

Keywords: Energetic materials, PETN-I and $\beta$-HMX, density-functional theory, equation of state, shock sensitivity, shear stresses

PACS: 31.15.Ar, 31.15.Ew, 31.70.Ks, 62.50. $+\mathrm{p}, 64.30 .+\mathrm{t}$

\section{INTRODUCTION}

One of the goals of energetic materials (EMs) research is to obtain accurate equations of state (EOS) for several important classes of EMs. Although a number of theoretical and experimental studies have been performed to obtain hydrostatic EOS for several $\mathrm{EMs}^{1-9}$, there is an urgent need to extend isotropic EOS to include a description of the materials response upon uniaxial compression. The uniaxially compressed state of the crystal is directly related to the state that a crystal experiences upon shock loading. The shear stresses upon uniaxial compression are of particular interest because they are usually considered to be the driving forces behind plastic deformations in crystals and the sources of mechano-chemical reactions behind the shock wave front.

Anisotropic response to plane shock wave loading was observed in the experiments on PETN by J. J. Dick ${ }^{10,11}$. Detonation at relatively low input stresses and run distances was observed for shocks delivered in the $[110]$ and [001] crystallographic directions, yet detonation was not observed for shocks delivered in the [100] and [101] directions.

The goal of this work is to obtain highly accurate hydrostatic EOS for PETN-I and $\beta$ HMX and extend them to include uniaxial compressions in a wide range of compression ratios. We discuss the behavior of the shear stresses upon uniaxial compression and their potential importance in explaining the 
anisotropic shock sensitivity of PETN-I and $\beta$ HMX.

\section{COMPUTATIONAL DETAILS}

The density functional calculations were performed using the Vienna Ab-Initio Simulation Package (VASP) ${ }^{12}$. Special attention was paid to ensure high accuracy in the calculations. Test calculations were done to determine the types of density functionals and pseudopotentials, the value of the energy cutoff, and the density of k-point sampling within the Brillouin zone that are adequate to reproduce available experimental data on equilibrium crystal structures. From these tests, the Generalized Gradient Approximation (GGA) density functional theory (DFT) with the Perdew-Burke-Ernzerhof (PBE) functional, the soft projected augmented wavefunction (PAW) pseudopotential with a $400 \mathrm{eV}$ nominal cutoff, and a fixed Monkhorst-Pack grid corresponding to an average of $0.08 \AA^{-1} \mathrm{k}$-spacing at maximum compression have been chosen. An energy cutoff of $700 \mathrm{eV}$ was used, which yielded convergence better than $0.4 \mathrm{GPa}$ in pressure, $0.0015 \mathrm{eV} / \mathrm{atom}$ in energy, and $0.015 \mathrm{eV} / \AA \AA$ in forces

The equilibrium structures of PETN-I and $\beta$ HMX were calculated by relaxing all the degrees of freedom of the experimental structures ${ }^{4,13}$ including lattice parameters and atomic coordinates using the quasi-Newton method as implemented in VASP. For all relaxations in this study, the convergence for electronic steps was set to $10^{-6} \mathrm{eV}$, and relaxation continued until the maximum force on any atom within the unit cell was less than $0.03 \mathrm{eV} / \AA$.

Hydrostatic compression calculations were performed on single unit cells of PETN-I and $\beta$ $\mathrm{HMX}$ in the range of $\mathrm{V} / \mathrm{V}_{0}=1.06$ to 0.60 in increments of 0.02 , where $\mathrm{V}_{0}$ is the volume at zero pressure. Uniaxial compressions of both PETN and HMX were performed in the [100], [010], [001], [110], [101], [011], and [111] directions. For each compression direction, the calculated equilibrium unit cell was rotated such that the $\mathrm{x}$-axis corresponded to the direction of compression. Subsequently, the x-component of each lattice vector was scaled by $2 \%$ up to $\mathrm{V} / \mathrm{V}_{0}$ $=1.06$ and down to $\mathrm{V} / \mathrm{V}_{0}=0.70$.

\section{RESULTS AND DISCUSSION}

PETN-I. The calculated unit cell parameters were compared to experimental data from Olinger, Halleck, and Cady ${ }^{4}$. According to their work, the lattice constants $\mathrm{a}=\mathrm{b}=9.386 \AA$ and $\mathrm{c}$ $=6.715 \AA$. The lattice constants from this study (and the percent difference from experiment) were $9.617 \AA(+2.5 \%), 9.612 \AA(+2.4 \%)$, and $6.83 \AA(+1.7 \%)$ for $\mathrm{a}, \mathrm{b}$, and $\mathrm{c}$, respectively. The disagreement between DFT calculations and experiment for molecular crystals has been studied in several works $\mathrm{s}^{2,14}$ and has been attributed to poor description of van der Waals interactions by DFT.

The isothermal equation of state (EOS) from the hydrostatic compression simulations of this work was compared with the experimental data of Olinger et al. ${ }^{4}$, see Figure 1. Pressure, change in energy per atom, and band gap were plotted as a function of absolute volume in the range of $\mathrm{V} / \mathrm{V}_{0}=1.0$ to 0.60 . Good qualitative agreement was observed between the EOS calculated in this study and experimental data. For PETN-I, the elastic constants $\mathrm{C}_{11}, \mathrm{C}_{22}$, and $\mathrm{C}_{33}$ were calculated and compared with experiment ${ }^{15}$, see Table 1. Results on isotropic EOS and elastic

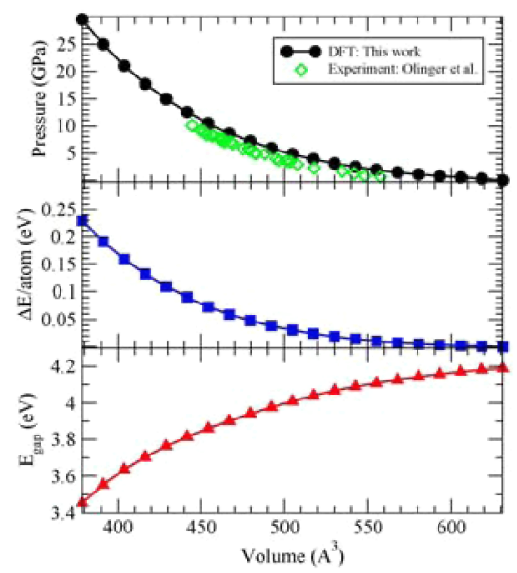

FIGURE 1. Hydrostatic EOS of PETN-I. 
TABLE 1. Elastic constants of PETN-I calculated in this work and compared with experiment ${ }^{15}$

\begin{tabular}{|c|c|c|c|}
\hline Study & $\mathrm{C}_{11}(\mathrm{GPa})$ & $\mathrm{C}_{22}(\mathrm{GPa})$ & $\begin{array}{c}\mathrm{C}_{33} \\
(\mathrm{GPa})\end{array}$ \\
\hline $\begin{array}{c}\text { Exp.: } \\
\text { Ref. 6 }\end{array}$ & 17.22 & 17.22 & 12.17 \\
\hline $\begin{array}{c}\text { This } \\
\text { work }\end{array}$ & $\begin{array}{c}18.25 \\
(+6.0 \%)\end{array}$ & $\begin{array}{c}18.5 \\
(+7.4 \%)\end{array}$ & $\begin{array}{c}14.5 \\
(+19 \%)\end{array}$ \\
\hline
\end{tabular}

constants have given us confidence that DFT gives a reasonable description of the compressed crystals despite the problem with van der Waals interactions.

In the case of uniaxial compressions of PETN-I, the change in energy per atom, longitudinal stress, band gap, and shear stresses were examined as a function of $\mathrm{c} / \mathrm{c}_{0}$. The shear stresses, which showed the greatest relative variation among the inspected properties, were calculated from the equation $\tau_{y(z) x}=\left(\sigma_{x x}-\sigma_{y y(z z)}\right) / 2$, where $\mathrm{x}$ indicates the direction of compression. Figure 2 shows the values of the shear stresses at uniaxial compression of $\mathrm{c} / \mathrm{c}_{0}=0.7$.

From the experiments of J. Dick ${ }^{10}$ on PETN$\mathrm{I}$, the [110] and [001] directions have exhibited a greater sensitivity to detonation via shock, and the [100] and [101] directions have displayed decreased sensitivity. It can be observed from Figure 2 that the shear stresses for the more sensitive directions, [110] and [001], exhibit

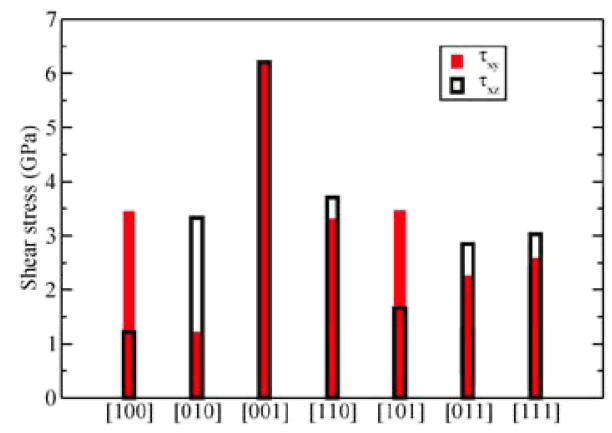

FIGURE 2. Shear stresses $\tau_{\mathrm{yx}}$ and $\tau_{\mathrm{zx}}$ at uniaxial compression 0.7 in PETN-I. greater values for both $\tau_{\mathrm{yx}}$ and $\tau_{\mathrm{zx}}$ than the directions of lower sensitivity, [100] and [101]. Reduced values of shear stresses and greater separation between $\tau_{\mathrm{yx}}$ and $\tau_{\mathrm{zx}}$ were observed in the less sensitive directions, [100] and [101]. Therefore, the suggestion ${ }^{10}$ that shear stress behavior is linked with sensitivity is supported by these results.

Non-monotonic dependence of shear stress upon strain was also observed for directions of decreased sensitivity. From these calculations and experimental data, we see that greater calculated values of both $\tau_{\mathrm{yx}}$ and $\tau_{\mathrm{zx}}$ upon uniaxial compression correlates positively with increased sensitivity in energetic molecular crystals.

$\boldsymbol{\beta}$-HMX. The calculated equilibrium lattice constants of $\beta$-HMX were compared with the experimental data from Choi and Boutin ${ }^{13}$. Their results were $\mathrm{a}=6.54 \AA, \mathrm{b}=11.05 \AA$, and $\mathrm{c}=$ $8.70 \AA$. The calculated values of the lattice constants from this work (and the percent difference from experiment) were $6.701 \AA$ $(+2.5 \%), \quad 11.347 \AA(+2.7 \%)$, and $8.910 \AA$ $(+2.4 \%)$ for $a, b$, and $c$, respectively. Again, overestimation of the lattice parameters was associated with the inability of DFT functionals to describe the significant intermolecular van der Waals interactions.

The calculated isothermal EOS was compared with the experimental data obtained by Gump and Peiris ${ }^{5}$ and Yoo and Cynn ${ }^{6}$, see Fig. 3.

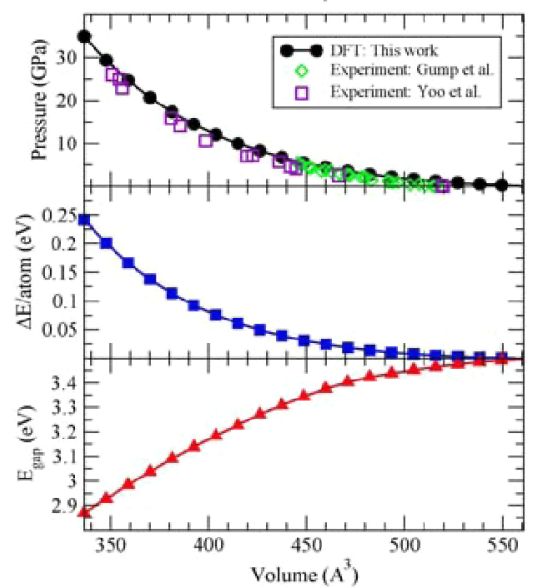

FIGURE 3. Hydrostatic EOS of $\beta$-HMX. 




FIGURE 4. Shear stresses $\tau_{\mathrm{yx}}$ and $\tau_{\mathrm{zx}}$ at uniaxial compression 0.7 in $\beta$-HMX.

As observed in PETN-I, DFT provides good qualitative agreement with the EOS data gathered from experiment for $\beta$-HMX.

The shear stresses for the uniaxial compressions performed on $\mathrm{HMX}$ at $\mathrm{c} / \mathrm{c}_{0}=0.7$ were plotted in Fig. 4. Greater magnitudes of shear stresses, which corresponded to directions of greater sensitivity in PETN-I, were present in the directions [110] and [010]. Consequently, if the trend found for PETN-I is maintained for $\beta$ HMX, then greater sensitivity to initiation is expected in these directions.

\section{CONCLUSIONS}

The energetic material crystals PETN-I and $\beta$-HMX were studied using first-principles density functional theory. Good agreement was observed between available experimental data and calculated equilibrium structures, hydrostatic equations of state, and elastic constants. The errors were attributed to poor description of van der Waals interactions by DFT. The inclusion of van der Waals interactions will be the subject of future work.

The isotropic EOSs have also been extended to include uniaxial compressions PETN-I and $\beta$ HMX in the [100], [010], [001], [110], [101], [011], and [111].

Upon comparison of the simulated uniaxial compression data with experimental information on the anisotropy in sensitivity to shock-induced detonation for PETN-I, a correlation between shear stresses and sensitivity was suggested. The magnitudes of the calculated shear stresses were greater for the more sensitive directions, [110] and [001], than for the less sensitive directions, [100] and [101]. Extrapolating this trend between calculated shear stresses and known sensitivity in PETN to our calculated results for $\beta$-HMX suggests that for $\beta$-HMX the [110] and [010] directions could be more sensitive to initiation,

\section{ACKNOWLEDGEMENTS}

The work at USF was supported by the Office of Naval Research (ONR) through the Naval Research Laboratory (NRL). The work at NRL was also supported by ONR both directly and through NRL. M. Conroy thanks the organizing committee of SCCM 2007 (Ricky Chau) for the travel support to attend SCCM 2007 in Hawaii.

\section{REFERENCES}

1. Sorescu D. C., Rice B. M., and Thompson D. L., J. Phys. Chem. B 103, 6783 (1999).

2. Byrd E. F. C., and Rice B. M., J. Phys. Chem. C 111, 2787 (2007).

3. Liu H., Zhao J., Wei D., and Gong Z., J. Chem. Phys. 124, 124501 (2006).

4. Olinger B., Halleck P.M., and Cady H.H., J. Chem. Phys. 62, 4480 (1975).

5. Gump J. C., and Peiris S. M., J. App. Phys. 97, 053513 (2005).

6. Yoo C., and Cynn H., J. Chem. Phys. 111, 10229 (1999).

7. Gan C., Sewell T. D., and Challacombe M., Phys. Rev. B 69, 035116 (2004).

8. Brand H. V., J. Phys. Chem. B 109, 13668 (2005).

9. Sewell T. D., Menikoff R., Bedrov D., and Smith G. D., J. Chem. Phys. 119, 7417 (2003).

10. Dick J. J., Appl. Phys. Lett. 44, 859 (1984).

11. Dick J.J., J. Appl. Phys. 76, 2726 (1994); 81, 601 (1997).

12. Kresse, G.; Furthmuller, J. Vienna Ab-Initio Simulation Package (VASP)

13. Choi C. S., and Boutin H. P., Acta Crystallogr., Sect. B 26, 1235 (1970).

14. Byrd E. F. C., Scuseria G. E., and Chabalowski C. F., J. Phys. Chem. B 108, 13100 (2004).

15. Winey J. M., and Gupta Y. M., J. App. Phys. 90, $1669(2001)$. 